\title{
An Adaptive Cruise Control Model based on PDLCA for Efficient Lane Selection and Collision Avoidance
}

\author{
Khawaja Iftekhar Rashid \\ Department of Computer Science \& Technology \\ Dalian University of Technology, (DUT) \\ Dalian, Liaoning, Post (116024), P.R. China \\ https://orcid.org/0000-0001-5003-7969 \\ Ding Nan \\ Department of Computer Science \& Technology \\ Dalian University of Technology, (DUT) \\ Dalian, Liaoning, Post (116024), P.R. China
}

\author{
Muhammad Tahir \\ School of Software Technology \\ Dalian University of Technology, (DUT) \\ Dalian, Liaoning, Post (116621), P.R. China \\ Anil Ahmed \\ Department of Computer Science \& Technology \\ Dalian University of Technology, (DUT) \\ Dalian, Liaoning, Post (116024), P.R. China
}

\begin{abstract}
Todays, safe and sans collision free traveling is essential in the present World. Because of the particular needs required by different usages of Vehicular Ad Hoc Networks (VANETs), mainly in evolving lane, outlining a constant Collision Avoidance (CA) and securely lane changing has turned into the foundation of controlling the vehicle in the dense environment. In our system, we proposed efficient lane selection and collision-free vehicle transportation system in the light of patience, speed and the distance between all types of vehicles. To maximize the efficiency of the existing transportation infrastructure and reduce collision, it is necessary to improve safety and communication in all vehicles. In this paper, we first define the patience based method and simulate model for safety and efficiency of traffic flow using the game theoretical technique. Then we have improved a new algorithm for lane selection and collision control using distance and speed of all vehicles gather from the vehicular communication. After two different methodologies for lane change and collision-free driving are compared like; random patience based method and car following method, based on different traffic density. In the part of contribution as an outcome, our experimental simulation setup is executed on highway road traffic scenarios to demonstrate the capacity of the all type of vehicles to select best-fit lane, identify collision, and explore around them to maintain the safe distance. Finally, it is concluded that our proposed algorithm outperforms in lane selection and collision avoidance to satisfy safety and efficiency in the Vehicular Ad Hoc Networks.
\end{abstract}

Keywords-Automatic vehicle; collision avoidance; lane selection; patience; threshold; traffic density

\section{INTRODUCTION}

Vehicle-to-Vehicle communications (V2V) provide the opportunity to establish the connection between vehicles through the recent advancement in communication technologies. The experiments in planning a Collision Avoidance (CA) and the framework are adjusting the viability of maintaining a strategic distance from collisions versus the danger of false alerts. The assignment of a CA is the framework to track objects of potential collision hazard and to maintain the threshold for avoiding the collision. Vehicle applications specifically display few difficulties; dense traffic causing complex situations with many moving articles; minimum distance sensors and computational units must be utilized [1], [2]. According to author's focus on how to make decisions based on uncertain estimates and in the presence of multiple obstacles. Timely detection of other vehicles in the region of outrageous significance to help avoid an accident and potential loss of human life, and traffic jams. Driver distractedness, weakness, and juvenile conduct are the fundamental variables causing street mishaps [3]. As indicated by the National Highway Traffic Safety Administration (NHTSA), that almost $25 \%$ of police-announced accidents embroil some sort of driver distractedness the driver may be diverted, exhausted, snoozing or somewhere out in dreamland [4].

Acceleration and lane-change decisions are drivers' main operational and tactical decisions. Lane change is considered as a standout amongst the most difficult driving moves to comprehend and to foresee, and with related driving choices are frequently observed as a noteworthy wellspring of collision [5]. Among current examinations, a large portion of lane change models in light of vehicle kinematics estimate that when the neighbor vehicle changes lane, the accompanying vehicle of target lane keeps uniform movement [6], [7]. Adaptive Cruise Control (ACC) is typical and surely understood driving assistant system. By depending on a frontal radar or a 'LIDAR sensor', it can automatically adjust speed to keep up a protected progress distance between the vehicles in a similar lane. A few automakers have just presented highlights, for example, the ACC, in their top-of-the-line cars and some are seeking after research to incorporate the ACC, framework and collision warning or collision avoidance frameworks in different -situations. These frameworks utilize the basic plan that announces the target vehicle as the nearest one right now in subject's lane. Significant disadvantages coming about because of these fundamental methods are the failure to identifying vehicle on other lane and performing sufficient 
response ahead of time and the restricted execution in high arch location [8], [9].

A few works have been done in the field of ACC, for instance in the field of automatic braking, parking, forthcoming collision control, automatic crossing point assistance, automatic lane change and lane keeping framework that take after the speed of the lead vehicle. Apart from these social or legitimate issues while managing ACC, with VANET, obviously there is an achievement for enhancing wellbeing if the frameworks can act quickly and successfully. However, it should be notice that the highly complex quality associated with these situations, these frameworks request a high measure of vehicles data and the environment [10]. Congestion arises in the network when traffic increase and stuck in the lane instead of changing their lanes. ACC, assistances to enhance driver support and increase highway limit. Likewise; by applying automatic vehicle following framework, the collision between vehicles can be sufficiently decreased. Regardless, the greater part of them is executed in straight forwarding situations. Broad research has been done on the longitudinal speed control of vehicles. In this research paper; we compared Random Patience based Lane Change Algorithm (RPLCA), and Lane Change Model based on Car-Following Behavior (LCMCFB), that help the autonomous vehicle to change lanes in different situations and avoid the collision. Based on this concept we present our new idea of lane change by setting a threshold based on the distance between vehicles and different values of patience. Moreover, we use the game theoretical techniques based on zero-sum non-cooperative game theory under incomplete information. The type of game represents lane changing decisions that are made when the driver has partial information about other driver's decision. Rest of our paper deals as follows: In Section II, we are providing the overview of some work related to our experimental design which is based on collision avoidance methodology as well as patience based approach. Section (III) provides simulation scenario and mentions proposed method briefly. In Sections (IV) and (V), we discuss the experimental environment and discuss the result respectively. In the end, we conclude our remarks in Section (VI).

\section{RELATED WORK}

VANETs, apply numerous channels, includes Control Channels $(\mathrm{CCH})$, and Service Channels $(\mathrm{SCH})$, to give open street wellbeing service and enhanced comfort and productivity of driving. $75 \mathrm{MHz}$ of DSRC, the range at $5.9 \mathrm{GHz}$ extraordinarily assigned by FCC that is uniquely intended for vehicular correspondence is separated into seven equivalent channels called $\mathrm{CCH}$ and $\mathrm{SCH}$ [11]. Subsequently, the collision may occur when a vehicle changes its lane without having proper information related neighbor vehicles. ACC is a procedure used to control the speed of a vehicle to maintain safe distance from the leading vehicles. The essential point of this strategy is to give a reasonable alleviation to the driver. Locally available sensors help to control speed and maintain distance with the help of data received from Road Side Infrastructure (RSI), satellites and messages broadcast from surrounding vehicles. Some of the application installed in vehicles to detect leading vehicle which are mention here. A laser-based framework couldn't recognize leading vehicle in foggy climate or when the vehicle is muddy. Other techniques including single radar-based framework and binocular computer vision framework in view of the front camera are used in vehicle detection [12], [13].

\section{A. Collision Avoidance Methods}

Several methods have been introduced in VANET to avoid collision between vehicles to protect lives and efficient driving. Here we are discussing some techniques related collision avoidance by different researchers. Alessandro Colombo et al. consider the issue of impact control at the intersection point. The author considers it as an NP-hard issue and is around illuminated in this paper, by receiving concentrated approach in light of supervisor having provable error bound [14]. The supervisor depends on a hybrid approach that utilizes a dynamic model of the vehicles and occasionally tackles a scheduling issue. The supervisor act as a channel between a coveted inputs forms from driver side and the physical framework. This structure is effortlessly combined with different controllers, acting between the driver input and the supervisor, to seek after optional execution destinations inside the arrangement of safe control activities permitted by the supervisor.

An effort related collision avoidance and detection of independently moving vehicles are settled by receiving multioperator frameworks is discussed in [15]. Vehicles can commonly illuminate themselves about their objectives and goals for future development and subsequently can comprehend a conceivable collision significantly more productively than in the non-collaborative case. The proposed technique combine with a mind stunning conduct enabling it to consult with the workstation operators about items transportation, explore through the system to get to a specific workstation as quick as could be allowed, recognize and avoid collisions with different Automated Guided Vehicles (AGVs), and even powerfully identify and maintain a safe distance from hurdles on road.

Marwah M. Almasri et al. presents another solution for lane following and collision avoidance in the mobile robotic frameworks. The proposed system depends on the utilization of minimal effort infrared sensors and includes the sensible level of estimations, with the goal that it can be effectively utilized as a part of ongoing control applications. This technique demonstrates that the robot has been effectively followed extremely congested curves and encounter any obstacle on its way [16]. Above work is firmly identified with our simulation related collision recognition and lane change control. In our collision avoiding scenario we consider above work in term of the vehicular environment. Where we get the location from beacon messages and compute the distance between the vehicles.

\section{B. Patience based Method}

In our simulation, we dynamically choose the lane that is suitable for safe and efficient transport on road by collecting data of close-by vehicles, their speed, distance and number of vehicles in various lanes. These features help us to take the correct decision. Dissimilar to [17], we better utilize patience 
level to change lane (moving from the congested lane to the one having low traffic). The factor of patience has been discussed by few researchers. We are reviewing some of them in this section. Patience parameter was presented in [18]. While going astray from the favored speed vector in protocol constrained autonomous collision avoidance algorithms permitting a weighted balance of a lane selection and speed change. The patience parameter and suggested measurements of research permit autonomous collision avoidance with surrounding vehicles to maintain a safe distance from the collision. The patience parameter is presented for autonomous vehicles in sea scenario utilizing multi-target advancement with a non-parallel collision avoidance space involving distance factor. The experiment has performed in light of various values of the patience from 1-99. In our simulation, we utilize patience parameter and set the value to maximum for all the vehicles, and calculate distance and speed to select lane and speed. We will prove that the speed of vehicles and change lane incredibly influence the collision and lessen blockage and help the system to work efficiently. We compare two algorithms and based on above mention factor presents our algorithm "Adaptive Cruise Control Patience and Distancebased Lane Change and Collision Avoidance Algorithm (ACCPDLCA)" to improve traffic efficiency and avoid the collision. ACC can just reduce the speed of the vehicle and help drivers to avoid the collision by maintaining the safe distance from the leading vehicle and help him to change speed.

\section{PROPOSED WORK}

\section{A. Traffic Scenario}

The substance of our exploration in view of Cooperative awareness messages - According to [19], the cooperative awareness well known as beacon message contains the information related current status, geolocation and service announcements to/from those vehicles. This information related geolocation is further used in our research in calculating the distance between the vehicles. For safety driving, it is essential to have the location of surrounding vehicles to calculate the distance and make a decision whether to apply break, slow down speed, speed up or change the lane.

In aforementioned work based on patience level, the lane change factor of a vehicle depends upon the level of patience parameter, when the vehicle applies break each time its patience values reduce by 1 and speed reduced with the factor of deceleration. We have simulated different scenarios on the basis of different variables involved likewise acceleration, deceleration, patience value, and vehicles density. When it reaches to 0 , the vehicle changes its lane and shift to one of the closest lanes. In a high-density traffic environment when a vehicle changes its lane only based on patience level, chance of collision increase. For example, an engine failure or a mishap involving two or more vehicles can lead to a traffic jam. For this scenario, the objective is to build the vehicle efficiency for singular vehicles. Moreover, contingent upon various factors, for example, the significance of the accidental area, the vehicle framework may figure and recommend elective lanes to an expansive arrangement of vehicles considering a more extensive perspective of the movement requests keeping in mind the end goal to reduce the effect of this accident to areas not near to that instance. For this scenario, the objective is to expand the general transport productivity. Another issue is that when a vehicle changed its lane the value of patience is again set to maximum to avoid frequent lane change. Traffic scenario for lane selection and change is illustrated in Fig. 1.

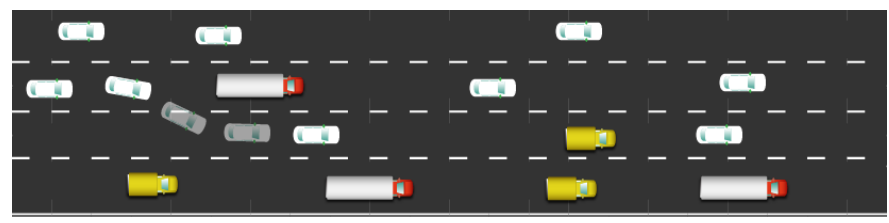

Fig. 1. The Road Traffic Scenario based on all types of vehicles when a high-speed vehicle stuck in the lane and change the lane from the best available lane.

\section{B. Proposed Simulation Model}

In our proposed solution we set the different threshold to change lane with proper information and having the best available solution that we achieved by using Bayes probability algorithm in our simulation. First, we check the traffic density of each lane and the speed of neighbor vehicles before lane change. Second, we start to make the decision of lane change based on distance and speed of the vehicle ahead. The third one we used Bayes probability theorem to decide whether to change lane or remain intact with the speed of the vehicle ahead. Increase in the density of traffic reduces the overall speed of vehicles as well as increase the chance of collision. To achieve maximum speed on-road vehicles choose a lane having low traffic with maximum speed. During this process, several collisions occur. To tackle the problem-related lane change and increase the overall speed of the vehicles we proposed an algorithm based on patience value and distance between the vehicles.

Another push to control blockage and increase the speed of the vehicles to run smoothly and efficiently we used the patience factor. However, the preference and threshold of patience are different in our simulation than the one used in the ocean based technique as we discuss in Section II. The equation to detect threshold for patience is given below.

$$
p_{\text {threshold }}=\frac{p_{\text {Max }} * v_{x y}}{\mathrm{~L}}
$$

Where $\mathrm{p}$ is the patience, $\mathrm{v}_{\mathrm{xy}}$ is the speed of the vehicle and $\mathrm{L}$ represents the number of lanes. After calculation of the thresholds, after that, we decide to change the lane of the vehicles along considering other factors. When the lane of the vehicle changed the patience value is again set to max. An equation to compute the safety threshold of distance is as under.

$$
d_{\text {threshold }}=\alpha *\left(\text { sizec }_{\text {ahead }}+\text { sizec }_{\text {self }}\right)
$$

$\alpha$ should lie in between $[0.5,1]$. If sizec $_{\text {ahead }}>$ sizec $_{\text {itself }}$ then the value of the constant should be equal to 1 for the lane change to avoid the collision. If sizec $_{\text {ahead }} \leq$ sizec $_{\text {itself }}$ then the constant value should be set as 0.5 . If the value of constant is less than 0.5 then chance of collision increased. Size ahead $_{\text {and }}$ 
size $_{\text {itself }}$ both are variables, used to store values of the size the vehicles.

Before settling on any choice on the premise of information and earlier outcomes we initially check the possibility of collision. On the premise of those probabilities, we choose the proper lane and distinctive limits to diminish the danger of collision. Utilizing these probability estimations we plan structure for performing simulations. To stay away from collision we should have former data of the vehicular network. On the premise of information obtained from the vehicular network we characterize the probabilities and change the lane and speed of the vehicle to avoid collision. In Bayesian probability hypothesis, one of these "instances" is the assumption, $\mathrm{H}$, and the other is information, $\mathrm{D}$, and we wish to judge the relative truth of the theory given the information.

In our research, we have an arrangement of variable information $\mathrm{D}=(\mathrm{D} 1, \mathrm{D} 2, \mathrm{D} 3, \ldots . . \mathrm{Dn})$ the reasons for collision $\beta$ and an arrangement of feasible lane $\mu$. As indicated by Bayesian control we utilize following recipe to choose the following move of the vehicle about the lane change.

$$
P(\mu \mid D)=\frac{\mathrm{P}(\mathrm{D} \mid \mu) \mathrm{P}(\mu)}{\mathrm{P}(\mathrm{D})}
$$

Since the data related lane $\mathrm{P}(\mu)$ is earlier access to the vehicle when it joins the network. $\mathrm{P}(\mathrm{D})$ is likewise obtained by summing up $\mathrm{P}(\mathrm{D} \mid \mu)(\mathrm{P} \mid \mu)$. The term $\mathrm{P}(\mathrm{D} \mid \mu)$ is the probability capacity and it evaluates the likelihood of the examined information acquired from the data received from various sources for our scenario beacon messages, GPS and so forth. $\mathrm{P}(\mu \mid \mathrm{D})$ is posterior. To maintain a strategic distance from the collision between vehicles one must realize what caused the collision. From (4) we induce the best arrangement of causes to clarify a given bit of information, as a rule, includes boosting the posterior over sources.

$$
\hat{\beta}=\frac{\arg \max _{\beta} P(\beta \mid D, \mu) P(\beta \mid \mu)}{P(\beta \mid D)}
$$

In (4) we utilize Nash Equilibrium in light of the fact that in a Nash balance, every vehicle's decision of activity is the best reaction to the moves really made by his adversaries. Subsequent to ascertaining the qualities and deciphering the accessible information it is important to choose the best lane to limit the hazard. Particularly in the dense network, it is hard to change lane. Utilizing Bayes likelihood hypothesis we assess the best reasonable lane from all the access lanes. Our activity is to choose the best lane $\mu$ from the information interpreted. To benefit the best lane we need to choose the lane having the best estimation of $\mu$. Mathematically it is expressed as

$$
\hat{\mu}=\arg \max _{\mu} P(\mu \mid D)
$$

\section{Game Theoretical Method}

Adaptive Cruise Control (ACC) is a discretionary voyage control framework for roadside vehicles that naturally alters the vehicle speed to maintain safe distance from the leading vehicles. Control depends on sensor data from locally available sensors. ACC innovation has broadly viewed as a key segment of any future ages of intelligent autos. They affect driver wellbeing and accommodation and also expand the limit of roads by maintaining safe distance among the vehicles and diminishing driver's mistakes. The intelligent control of vehicles is one of the present difficulties of intelligent transport frameworks. The utilization of Artificial Intelligence strategies to vehicle frameworks empowers driving help frameworks to interface with nature. Previous work-related ACC can help to avoid front-end collision based on data interpreted by the sensors onboard.

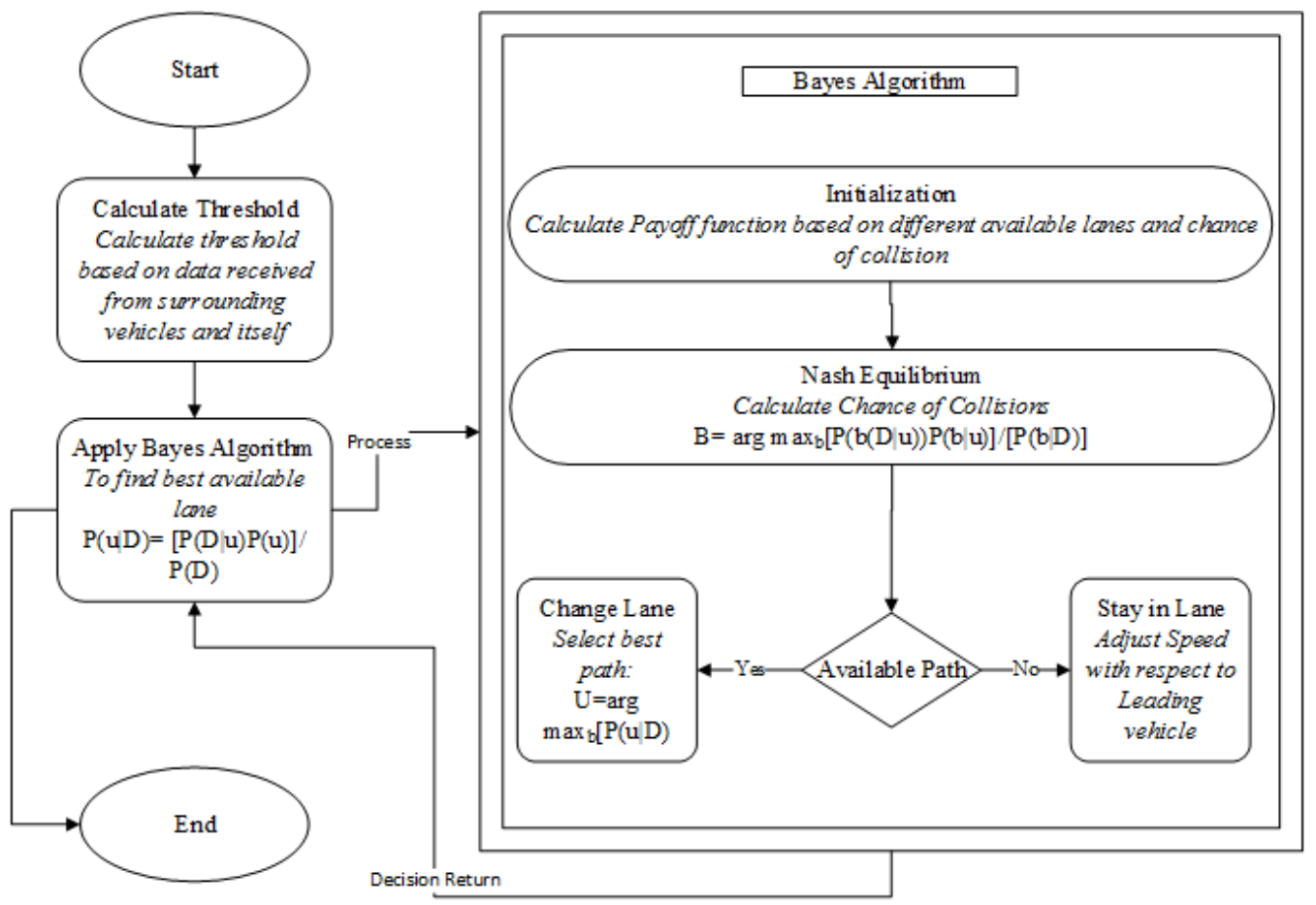

Fig. 2. A flow diagram of lane selection process for ACCPDLCA. 

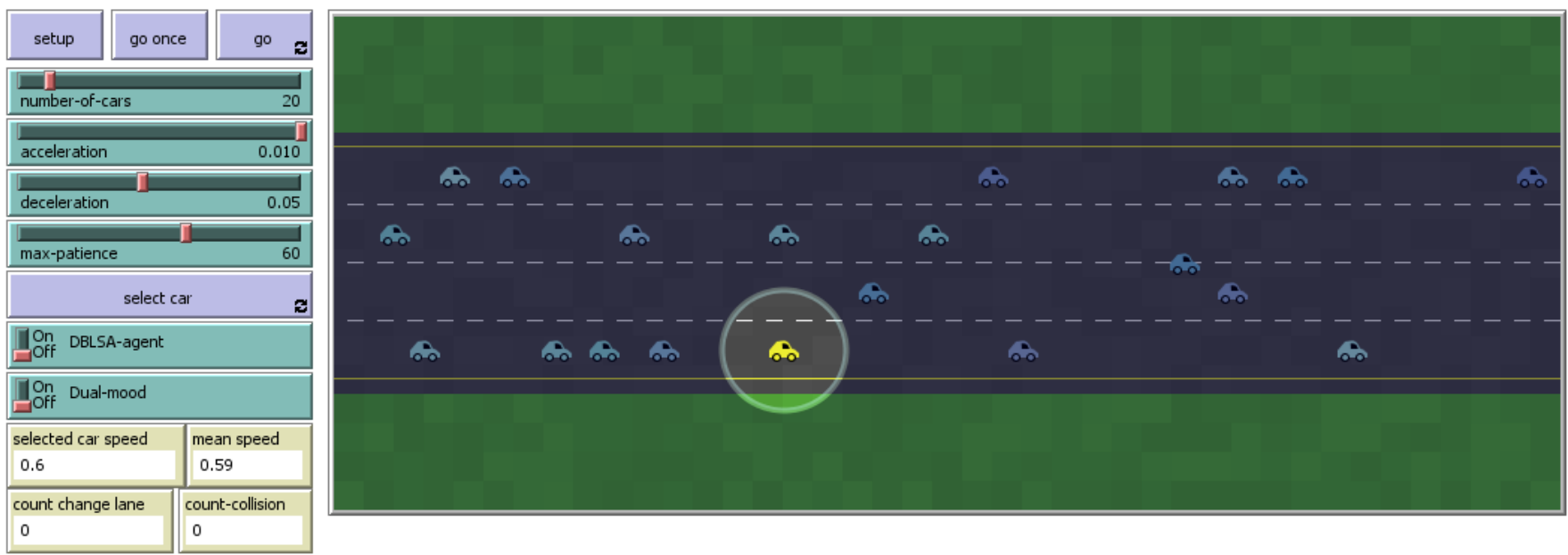

Fig. 3. Proposed simulation setup executed on low-density traffic for high-way road.

In our research we are dealing with lane change scenario, having distinctive extra highlights moreover vehicle ad-hoc correspondence with the surrounding vehicle to consider their speed, area, and other essential messages to guarantee wellbeing and effectiveness on road. VANET, correspondence helps us to gather relative information from different vehicles in the system utilizing reference point messages. With the assistance of these messages, we compute the general proficiency of the system. Area and speed of encompassing vehicle is to execute choice made by the vehicle. The framework of our experimental simulation is shown in above Fig. 2.

Let a vehicle $c_{i}$ moves with a speed of $v_{i}$ having patience value $p_{i}$ at time $t$ in lane $L_{i}$ following vehicle $c_{j}$ with a distance d. Utilizing ad-hoc correspondence between vehicles it will likewise assemble related data communicate much of the time from the encompassing vehicles. All these information stored in a tabular form maintained by each node. These beacon messages enable them to know current movement and speed of its surrounding vehicles. It persistently computes the distance between the vehicles ahead and furthermore has the data about their speed. At the point when the vehicle approaches the base distance criteria $d_{\text {threshold }}$ it will begin decelerating with the factor of deceleration. The patience esteem diminishes much of the time with deceleration. Meanwhile, when patience esteem $p_{i}$ approaches $p_{\text {threshold }}$ esteem it will then execute next step. At the point when the distance is way to deal with least limit and the patience likewise fulfill its condition then the estimation in light of the accessible information will perform through game theory approach. At that progression, we ascertain the most effective way and reasonable scenario for the vehicle. We simulate our experiment on different values of patience and traffic density and conclude the results in Section V.

\section{EXPERIMENTAL SETUP}

The experiment is performed in NetLogo software version v 6.0.2 [20], designed by Uri Wilensky. It shows programming ideas utilizing specialists as vehicles, patches, links and the spectator. We perform our experiments in highway scenario, by considering all types of the traffic density. We perform simulations in low density, medium density, and high-density traffic condition. The number of vehicles will be chosen progressively. Additionally, acceleration and deceleration are likewise factor and are also accessible at User Interface (UI), for dynamic determination. The patience level is also dynamic. We have performed several experiments with different level of patience from one to hundred and study the behavior of vehicles with respect to speed and collision avoidance. Simulation environment in Net Logo with low-density traffic is shown in Fig. 3.

Here we present another idea of ACC, Patience and Distance-based Lane Change Algorithm (ACCPDLCA), based on the game theory approach. It takes the position of the leading vehicles to set the speed and distance of every single close-by vehicle to measure collision probability. ACCPDLCA, based on a game theory proposed a few principles as per which every vehicle act. To accomplish our destinations we infer any forecasts whatsoever in various circumstance by describing how vehicles carry on, what their targets are and how guidelines of the diversion they attempt. Simulation results that vehicle knows the procedures accessible to every vehicle have finished and reliable preferences over conceivable results and they know about those preferences. Besides, they can decide the best lane for themselves and faultlessly execute it. On the off chance that a result isn't a Nash equilibrium, at that point no less than one of the vehicles isn't best reacting, and at some point or another a vehicle in that part will happen to arrive on a higher activity which will then be received by the vehicles a while later. This helps vehicles to choose lane having maximum speed. The decision is based on the minimum threshold value of patience and distance between the vehicles.

The number of accessible vehicles in a lane is consistently refreshed and was shown in a table. While in the dual mood we use two different algorithms run at a time on different vehicles to compare the effect of communication on the network. We can select the vehicle dynamically and observe its behavior in different frames. Here we apply ACCPDLCA on the selected vehicle while all the other vehicles in the network work base on ACC technology. In given simulation minimum speed of the vehicle is 0 , i.e., complete break while we set start speed equal to 0.5 . After that speed increased randomly up to 1.2 . 


\section{A. Simulation Parameters}

We performed the simulation in NetLogo default setup with four number of lanes. Each of the underneath simulations keep running for 20 and 40 minutes. Therefore, we ponder the informational index of this renovation made on each step. Results are placed in the spreadsheet and also we obtain in tabular form. For each activity condition, we have an informational index of 648,540. General we interpreted $2,594,160$ of informational collections during simulation. Result generated based on simulation data is discussed in Section V. In Table I, we represent out simulation parameters.

TABLE I. REQUIREMENTS OF SIMULATION PARAMETERS

\begin{tabular}{|l|l|}
\hline Simulation Tool & NetLogo \\
\hline Traffic Environment & $\begin{array}{l}\text { Low, Medium, High, Extreme } \\
\text { Dense }\end{array}$ \\
\hline Traffic Density (vehicle/lane) & variable \\
\hline $\begin{array}{l}\text { Average speed (at time of } \\
\text { joining) }\end{array}$ & 0.5 \\
\hline Max Speed & 1.2 \\
\hline Patience & $\begin{array}{l}10,20,30,40,50,60,70,80,90, \\
100\end{array}$ \\
\hline Acceleration & $0.002,0.006,0.01$ \\
\hline Deceleration & $0.02,0.06,0.1$ \\
\hline Simulation Time (sec) & 1200,2400 \\
\hline Data Set & At each step \\
\hline
\end{tabular}

\section{FINAL RESULTS AND DISCUSSION}

We simulated several experiments to prove that our algorithm help vehicles to select the better lane and enhance the speed by maintaining a safe distance from traffic congestion and collision between vehicles. We compared our algorithm with Random Patience based Lane Change Algorithm (RPLCA), and with Lane Change Model based on Car-Following Behavior focusing on the kinematic behavior of the lane-changing vehicle in the process of accelerated lane change (LCMCFB). Table II has generated from low traffic condition. The simulation of our algorithm also included various patience values from 10-100. In low-density traffic lane change occur infrequently, however as indicated by the qualities we measure that if the lane change marvels happen just on the bases of patience with the random incentive at the joining of the system then it decrease the general speed of vehicles on road.

TABLE II. VALUES OBTAINED AT LOW-DENSITY TRAFFIC

\begin{tabular}{|l|l|l|l|l|l|l|}
\hline \multirow{2}{*}{$\begin{array}{l}\text { Patien } \\
\text { ce }\end{array}$} & \multicolumn{3}{|l|}{ Average Collision in } & \multicolumn{3}{l|}{ Average speed with } \\
\cline { 2 - 7 } & $\begin{array}{l}\text { RPL } \\
\text { CA }\end{array}$ & $\begin{array}{l}\text { ACCPDL } \\
\text { CA }\end{array}$ & $\begin{array}{l}\text { LCMC } \\
\text { FB }\end{array}$ & $\begin{array}{l}\text { RPL } \\
\text { CA }\end{array}$ & $\begin{array}{l}\text { ACCPDL } \\
\text { CA }\end{array}$ & $\begin{array}{l}\text { LCMC } \\
\text { FB }\end{array}$ \\
\hline 10 & 3 & 0 & 0 & 0.70 & 0.80 & 0.79 \\
\hline 20 & 3 & 0 & 0 & 0.72 & 0.78 & 0.79 \\
\hline 30 & 3 & 0 & 0 & 0.71 & 0.78 & 0.79 \\
\hline 40 & 3 & 0 & 0 & 0.73 & 0.80 & 0.80 \\
\hline 50 & 3 & 0 & 0 & 0.73 & 0.80 & 0.79 \\
\hline 60 & 3 & 0 & 0 & 0.73 & 0.78 & 0.80 \\
\hline 70 & 3 & 0 & 0 & 0.72 & 0.79 & 0.80 \\
\hline 80 & 3 & 0 & 0 & 0.75 & 0.80 & 0.80 \\
\hline 90 & 3 & 0 & 0 & 0.72 & 0.80 & 0.78 \\
\hline 100 & 2 & 0 & 0 & 0.74 & 0.78 & 0.79 \\
\hline
\end{tabular}

Unnecessary lane change condition can cause the collision with surrounding vehicles that travel at high speed in the destination lane. Fig. 4 represents the number of collisions on various level of patience. After performing several experiments with different level of patience ACCPDLCA performs better than RPLCA. When we increase the traffic on road up to medium level the odds of collision between the traffic increase uncommonly when the driver changes the lane without having any data related traffic scenario in different lanes and in addition to the speed of vehicles. In this circumstance, it is important to settle on the better choice. Values obtained in Table III validate the effect of lane change among medium level traffic. In this scenario when the number of lane change increases unnecessarily the general speed of the vehicles decrease. In such scenario, when a vehicle change lane the following vehicles have to applied break to maintain the safe distance to avoid the collisions and the patience of the following car decreased.

Unnecessary lane change decreases the overall speed of the vehicles. Comparison of these algorithms with respect to the number of collisions have illustrated in Fig. 5. ACC in view of PDLCA performs better in this scenario. In low and medium level density condition we see that general speed of vehicle remain close to each other in all algorithms. Also, collision avoidance in ACCPDLCA and LCMCFB are apparently equal. However, when the traffic increase, it is hard to oversee speed of the vehicles particularly when each vehicle has different patience level subsequent after joining VANET.

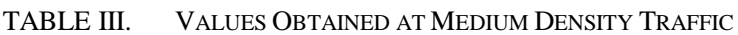

\begin{tabular}{|l|l|l|l|l|l|l|}
\hline \multirow{2}{*}{$\begin{array}{l}\text { Patien } \\
\text { ce }\end{array}$} & \multicolumn{3}{|l|}{ Average Collision in } & \multicolumn{2}{l|}{ Average speed with } \\
\cline { 2 - 7 } & $\begin{array}{l}\text { RPL } \\
\text { CA }\end{array}$ & $\begin{array}{l}\text { ACCPDL } \\
\text { CA }\end{array}$ & $\begin{array}{l}\text { LCMC } \\
\text { FB }\end{array}$ & $\begin{array}{l}\text { RPL } \\
\text { CA }\end{array}$ & $\begin{array}{l}\text { ACCPDL } \\
\text { CA }\end{array}$ & $\begin{array}{l}\text { LCMC } \\
\text { FB }\end{array}$ \\
\hline 10 & 33 & 3 & 4 & 0.36 & 0.69 & 0.50 \\
\hline 20 & 43 & 3 & 3 & 0.39 & 0.70 & 0.49 \\
\hline 30 & 36 & 3 & 4 & 0.41 & 0.68 & 0.68 \\
\hline 40 & 38 & 2 & 4 & 0.42 & 0.69 & 0.69 \\
\hline 50 & 38 & 4 & 3 & 0.43 & 0.68 & 0.68 \\
\hline 60 & 39 & 3 & 5 & 0.44 & 0.68 & 0.68 \\
\hline 70 & 40 & 3 & 4 & 0.43 & 0.68 & 0.68 \\
\hline 80 & 39 & 4 & 4 & 0.45 & 0.69 & 0.69 \\
\hline 90 & 40 & 3 & 5 & 0.45 & 0.68 & 0.68 \\
\hline 100 & 38 & 4 & 3 & 0.47 & 0.69 & 0.69 \\
\hline
\end{tabular}

TABLE IV. VALUES OBTAINED AT High-DENSITY TRAFFIC

\begin{tabular}{|l|l|l|l|l|l|l|}
\hline \multirow{2}{*}{$\begin{array}{l}\text { Patien } \\
\text { ce }\end{array}$} & \multicolumn{3}{|l|}{ Average Collision in } & \multicolumn{2}{l|}{ Average speed with } \\
\cline { 2 - 7 } & $\begin{array}{l}\text { RPL } \\
\text { CA }\end{array}$ & $\begin{array}{l}\text { ACCPDL } \\
\text { CA }\end{array}$ & $\begin{array}{l}\text { LCMC } \\
\text { FB }\end{array}$ & $\begin{array}{l}\text { RPL } \\
\text { CA }\end{array}$ & $\begin{array}{l}\text { ACCPDL } \\
\text { CA }\end{array}$ & $\begin{array}{l}\text { LCMC } \\
\text { FB }\end{array}$ \\
\hline 10 & 164 & 20 & 30 & 0.20 & 0.55 & 0.33 \\
\hline 20 & 196 & 24 & 28 & 0.22 & 0.54 & 0.33 \\
\hline 30 & 213 & 23 & 31 & 0.23 & 0.54 & 0.33 \\
\hline 40 & 219 & 23 & 28 & 0.24 & 0.54 & 0.33 \\
\hline 50 & 226 & 21 & 32 & 0.26 & 0.54 & 0.33 \\
\hline 60 & 224 & 20 & 31 & 0.25 & 0.55 & 0.33 \\
\hline 70 & 229 & 22 & 28 & 0.26 & 0.54 & 0.33 \\
\hline 80 & 228 & 24 & 34 & 0.26 & 0.55 & 0.33 \\
\hline 90 & 227 & 24 & 29 & 0.26 & 0.55 & 0.33 \\
\hline 100 & 233 & 22 & 30 & 0.26 & 0.55 & 0.33 \\
\hline
\end{tabular}


TABLE V. VALUES OBTAINED AT EXTREME High-DENSITy TRAFFIC

\begin{tabular}{|l|l|l|l|l|l|l|}
\hline \multirow{2}{*}{$\begin{array}{l}\text { Patien } \\
\text { ce }\end{array}$} & \multicolumn{4}{|l|}{ Average Collision in } & \multicolumn{3}{l|}{ Average speed with } \\
\cline { 2 - 7 } & $\begin{array}{l}\text { CAL } \\
\text { CACPDL }\end{array}$ & $\begin{array}{l}\text { ACA } \\
\text { CAC }\end{array}$ & $\begin{array}{l}\text { RPL } \\
\text { FB }\end{array}$ & $\begin{array}{l}\text { ACCPDL } \\
\text { CA }\end{array}$ & $\begin{array}{l}\text { LCMC } \\
\text { FB }\end{array}$ \\
\hline 10 & 690 & 289 & 678 & 0.11 & 0.30 & 0.19 \\
\hline 20 & 879 & 323 & 839 & 0.13 & 0.31 & 0.19 \\
\hline 30 & 981 & 184 & 850 & 0.14 & 0.31 & 0.18 \\
\hline 40 & 1008 & 294 & 781 & 0.14 & 0.31 & 0.19 \\
\hline 50 & 1070 & 265 & 769 & 0.15 & 0.31 & 0.19 \\
\hline 60 & 1128 & 259 & 774 & 0.15 & 0.31 & 0.19 \\
\hline 70 & 1163 & 302 & 639 & 0.16 & 0.31 & 0.19 \\
\hline 80 & 1151 & 278 & 536 & 0.16 & 0.31 & 0.19 \\
\hline 90 & 1167 & 276 & 666 & 0.16 & 0.31 & 0.19 \\
\hline 100 & 1173 & 240 & 734 & 0.16 & 0.30 & 0.19 \\
\hline
\end{tabular}

In Section III, we as of now specify that how factor estimation of patience impact the general speed of the vehicle and cause traffic block. In a high-density condition for the most- part patience value decreased to 0 of approximately every vehicle, since when we apply break or diminish speed with respect to the speed and conduct of the leading vehicle, subsequently patience value decreased to 0 . ACC in view of PDLCA help vehicles to sit tight for the others appropriate time to start lane change as portraying earlier. However, the performance of LCMCFB decreases as the traffic increase. Safety of vehicle during lane change scenario cannot only depend upon surrounding vehicle. A side collision occurs during lane change when we transfer to a lane in high-density traffic with proper information. Our proposed technique helps vehicles to remain in lane and continue proceeding onward with steady speed unless system generates safety message for transferring lane. These messages are generated as discussed in Section III above. Tables IV and V have been generated based on high-density and extreme high-density traffic from RPLCA, PDLCA, LCMCFB, on different patience esteem, respectively. Graphically represented in Fig. 6 and 7, respectively.

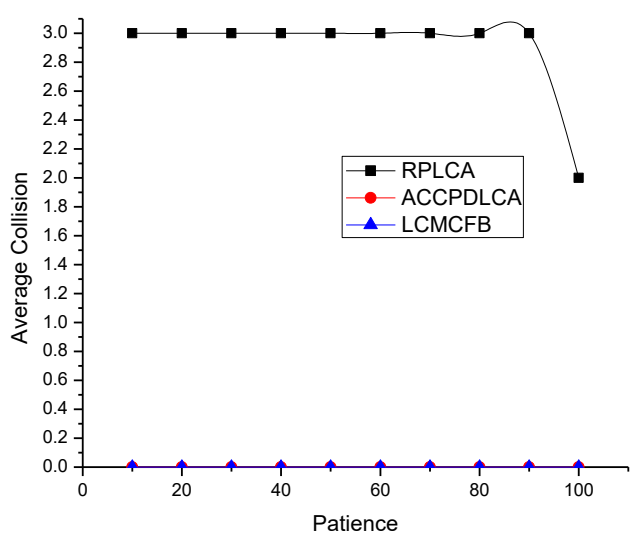

Fig. 4. Collision in low-density traffic environment.

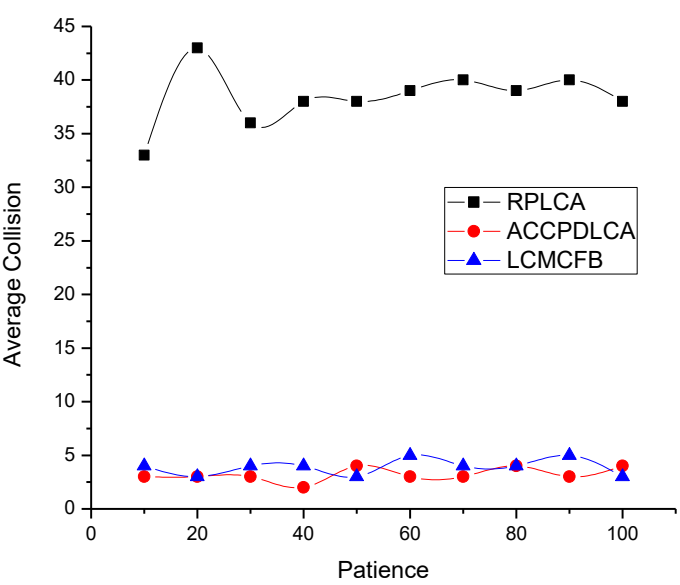

Fig. 5. Collision in medium density traffic environment.

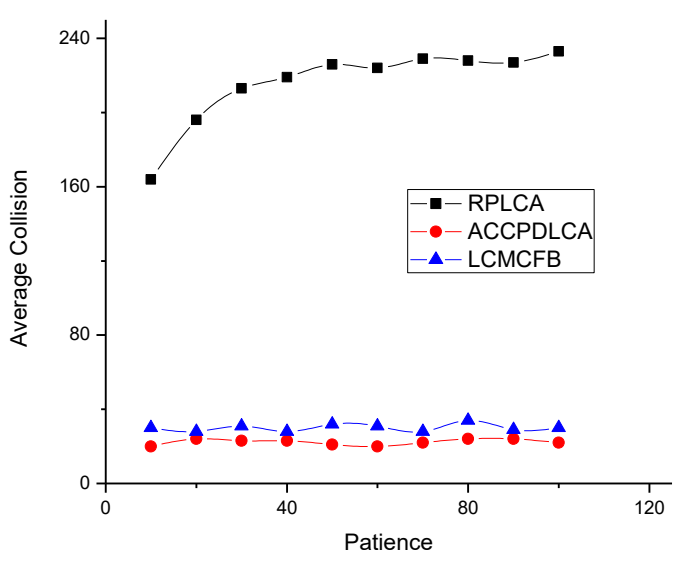

Fig. 6. Collision in high-density traffic environment.

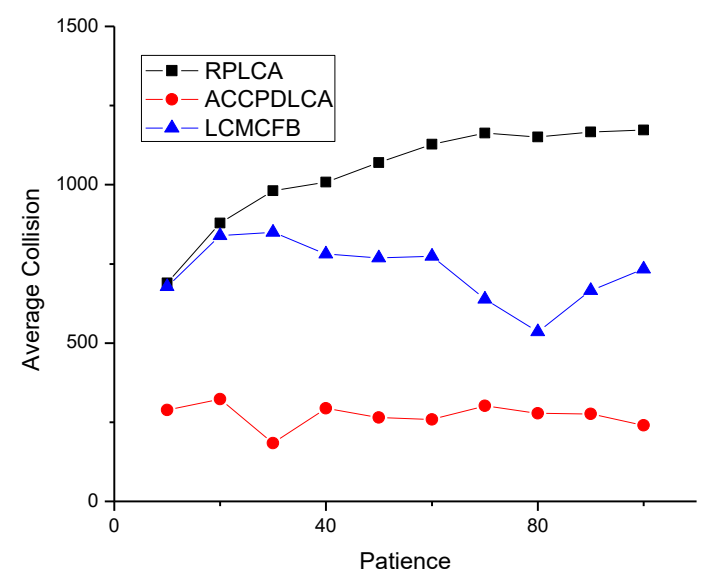

Fig. 7. Collision in extreme high-density traffic environment. 
In Fig. 8, we compare overall improvement in speed of vehicles in different density networks, using ACCPDLCA, LCMCFB, and RPLCA. The minimum speed of the vehicle can approach 0 while we set the starting speed 0.50 . The maximum speed touches 1.2. From the graph, it is dissipating that overall performance in term of speed in ACC with PDLCA is better than ACC without PDLCA (RPLCA). However, performance in term of efficiency (speed) of LCMCFB has also improved in low and medium density network. But in high and extreme dense environment LCMCFB shows lack of efficiency as well as safety.

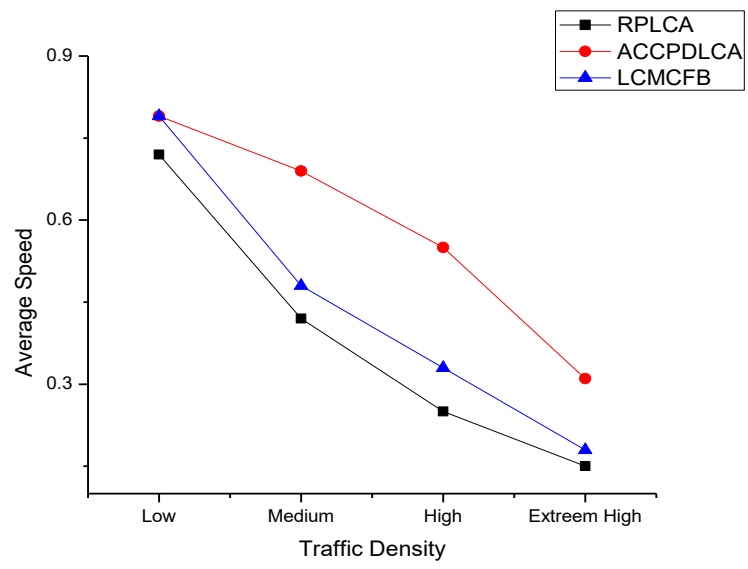

Fig. 8. Comparison of overall performance of vehicles in term of speed.

\section{CONCLUSION}

The strategy to enhance traffic efficiency in terms of speed and collision avoidance is discussed in this paper. The results obtained from our simulation verify that factor for patience with the coordinated effort of distance among vehicle performs better to enhance general execution of the system to keep away from a traffic jam in the dense environment. Our proposed solution Adaptive Cruise Control model based on Patience and Distance-based Lane Change and Collision Avoidance Algorithm (ACCPDLCA), performs better than Random Patience based Lane Change Algorithm (RPLCA), and with Lane Change Model based on Car-Following Behavior (LCMCFB).

The results shows that vehicles following ACCPDLCA have higher speed while RPLCA, decreases the speed and the efficiency of the traffic. Also, unnecessary lane change behavior in vehicles has been overcome by using Bayes algorithm and fixing the patience level to maximum when a vehicle joins the network in the specific area.

Maximum speed in high-density traffic are $0.55,0.33$, and 0.26 in $A C C P D L C A, \angle C M C F B$, and $R P L C A$, respectively. However, in extreme high-density environment where the distance between vehicles is lower than the threshold, our algorithm performs quite well as compared to others. The number of collision with respect to other two algorithms is also decreasing in number. Additionally, research about dense vehicular network will likewise be led.

\section{FUTURE SCOPE}

Safe and sound collision free traveling with high speed is the basic demand of the present World. The purpose of the algorithm is to suggest efficient lane selection and improve speed on the highway. Our algorithm is assisting drivers, by selecting the best suitable lane for safe and efficient driving. In the future, we will implement our proposed system in city traffic and multi-lane systems.

\section{ACKNOWLEDGMENT}

This work was supported by the National Natural Science Foundation of China No. 61471084, No. U1608253, and the open program of State Key Laboratory of Software Architecture No. SKLSA2016B-02.

\section{REFERENCES}

[1] J. Jansson, "Collision Avoidance Theory with Application to Automotive Collision Mitigation," Electrical Engineering, Link“oping University, 2005.

[2] A. K. G. G. W. T. Batra, "Collision detection system for vehicles in hilly and dense fog affected area to generate collision alerts," presented at the Issues and Challenges in Intelligent Computing Techniques (ICICT), Ghaziabad, India, 2014.

[3] P. S. S. L. Sagar D. Devagire, "Collision Avoidance Systems: A Review of Emerge Technology, and its Evolution," International Journal of Innovative Research in Science, Engineering, and Technology, vol. 5, 2016.

[4] A. M. L. X. T. B. Tang, "Vehicle Detection Techniques for Collision Avoidance Systems: A Review," IEEE Intelligent Transportation Systems Society, vol. 16, 24 March 2015.

[5] H. S. M. Alireza Talebpour, Samer H. Hamdar "Modeling LaneChanging Behavior in a Connected Environment: A Game Theory Approach," Transportation Research Procedia, vol. 21st International Symposium on Transportation and Traffic Theory 2015.

[6] F. R. PENG Jinshuan, SHI Leilei, ZHANG Qiong "Research of Driver s Lane Change Decision-making Mechanism," Journal of Wuhan University of technology, vol. 33, pp. 46-50, 2011.

[7] Y. H. WU Xiaorui, "A Lane Change Model with the Consideration of Car-Following Behavior," Procedia - Social and Behavioral Sciences, pp. $2354-2361,2013$.

[8] D. K. S. M. J. P. H. J. K. K. Yi, "Design of an Adaptive Cruise Control / Collision Avoidance with lane change support for vehicle autonomous driving," presented at the ICCAS-SICE, Fukuoka, Japan, 2009.

[9] D. K. S. M. J. P. H. J. K. K. Yi, "The Development of a Cooperative Heavy-Duty Vehicle for the GCDC 2011: Team Scoop," Intelligent Transportation Systems IEEE Transactions on, vol. 13, pp. 1033-1049, 2012.

[10] J. E. N. Felipe Jiménez, José Javier Anaya , Fernando García, Aurelio Ponz , José María Armingol "Advanced Driver Assistance System for road environments to improve safety and efficiency," Transportation Research Procedia, vol. 14, pp. 2245-2254, 2016.

[11] H. J. Reumerman, Z. Yupeng, G. R. Hiertz, G. S. Max, and L. Stibor, "Method for multi-channel resource reservation in a wireless mesh network," ed: Google Patents, 2012.

[12] D. A. F. Armin Askari, Alex A. Kurzhanskiy, Pravin Varaiya, "Measuring Impact of Adaptive and Cooperative Adaptive Cruise Control on Throughput of Signalized Intersections," 2016.

[13] G. W. Ziran Wang, Matthew J. Barth, "Developing a Platoon-Wide EcoCooperative Adaptive Cruise Control (CACC) System," presented at the IEEE Intelligent Vehicle (IV) Symposium, Redondo Beach, CA, 2017.

[14] A. Colombo and D. D. Vecchio, "Efficient algorithms for collision avoidance at intersections," presented at the Proceedings of the 15th ACM international conference on Hybrid Systems: Computation and Control, Beijing, China, 2012. 
[15] R. C. L. C.-W. Kuo, "Intelligent Seven-DoF Robot With Dynamic Obstacle Avoidance and 3-D Object Recognition for Industrial CyberPhysical Systems in Manufacturing Automation," Proceedings of the IEEE vol. 104, 24 March 2016.

[16] M. M. A. A. M. A. K. M. Elleithy, "Trajectory Planning and Collision Avoidance Algorithm for Mobile Robotics System," IEEE Sensors Journal vol. 16, June 15, 2016.

[17] S. Miyata, T. Nakagami, S. Kobayashi, T. Izumi, H. Naito, A. Yanou, et al., "Improvement of adaptive cruise control performance," EURASIP Journal on Advances in Signal Processing, vol. 2010, p. 295016, 2010.

[18] K. Woerner and M. Benjamin, "Autonomous Collision Avoidance Tradespace Analysis for High-Speed Vessels," in 13th International Conference on Fast Sea Transportation. Society of Naval Architects and Marine Engineers, 2015.

[19] X. W. Wenyang Guan, Ye Jin, "Channel congestion performance analysis for DSRC vehicle ad hoc network," presented at the 10th International Conference on Communications and Networking in China (ChinaCom), China, 2015.

[20] U. Wilensky. (1999). NetLogo. http://ccl.northwestern.edu/netlogo/. Center for Connected Learning and Computer-Based Modeling, Northwestern University, Evanston, IL.

\section{AUTHORS' PROFILE}

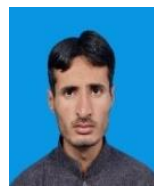

Khawaja Iftekhar Rashid received the B.Sc. and MCS degree from University of Punjab, Lahore, Pakistan and University of Azad Jammu and Kashmir, Muzaffarabad, Pakistan in 2012 and 2014 respectively. Currently pursuing his Master degree in Computer Science and Technology from Dalian University of Technology, Dalian, P.R. China. His research interests include vehicular networks, network information theory, wireless sensor networks, etc.

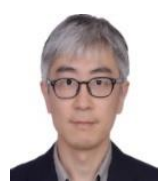

Nan Ding received the B.S., M.S., and Ph.D. degrees in Computer Science and Engineering from Dalian University of Technology, Dalian, P.R. China, in 1999, 2002, and 2006, respectively. He was a visiting scholar with the Electrical and Computer Engineering of University of New York University, U.S., from Sep 2013 to Sep 2014. He has been an Associate Professor in Computer Science and Engineering from Dalian University of Technology, Dalian, P. R. China since 2008. His research interests include vehicular networks, wireless security, wireless sensor networks, cache management, and distributed fault-tolerant computing.

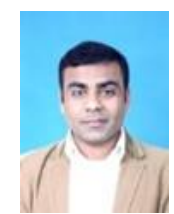

Muhammad Tahir received the BS (Hon's) degree in Software Engineering from University of Sindh, Jamshoro Pakistan in 2008 and Master of Engineering Degree in Software Engineering from CHONGQING University P.R. China in (2014). Currently, He is a Ph.D. Research Scholar in the School of Software Technology, Dalian University of Technology, P.R. China. His research interest includes Webbased Software defects prediction models, vehicular networks, Web-based tuning, IOT, Cloud Computing, SDN \& 5G.

Anil Ahmed received the B.S. degree in Computer

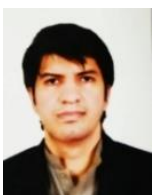
Science from Mirpur University of Science \& Technology, Mirpur, Pakistan in 2012. Currently, He is pursuing Master Degree in Computer Science and Technology from Dalian University of Technology, Dalian, P. R. China. His research interest includes Vehicular Networks, Deep learning, and CNN. 